\title{
Vertical forest structure characterization for the estimation of Above Ground Biomass: first experimental results using SAR vertical reflectivity profiles.
}

\author{
Astor Toraño Caicoya ${ }^{\text {a }}$, Florian Kugler ${ }^{\text {a }}$, Matteo Pardini ${ }^{\text {a }}$, Irena Hajnsek ${ }^{\text {a,b }}$, Konstantinos Papathanassiou ${ }^{\text {a }}$ \\ ${ }^{a}$ German Aerospace Center (DLR), Microwaves and Radar Institute (HR), POBOX: 1116, 82234, Wessling, Germany: \\ astor.toranocaicoya@dlr.de \\ ${ }^{b}$ ETH Zurich, Institute of Environmental Engineering, HIF D28.1, Schafmattstr. 6, CH-8093, Zurich, Switzerland: \\ hajnsek@ifu.baug.ethz.ch
}

\begin{abstract}
One common method to estimate biomass is measuring forest height and applying allometric equations to get forest biomass. However, changing forest density or forest structure bias the known allometric relations. Remote sensing systems like SAR or LIDAR allow to measure vertical forest structure. In this paper the value of vertical forest structure information for biomass inversion is investigated. First, vertical biomass profiles are calculated from forest inventory data. Then, a vertical structure descriptor based on Legendre polynomials is suggested and its sensitivity to biomass is evaluated. In a second step, this descriptor is used to describe SAR vertical reflectivity profiles. Then, a biomass estimation algorithm is developed. This is a case study based on inventory data from the Traunstein test site, a temperate mixed forest, located in the southeast of Germany.
\end{abstract}

Index Terms - Forest biomass, SAR reflectivity, vertical structure

\section{INTRODUCTION}

Forest above-ground biomass (AGB) stores an essential part of the terrestrial carbon and is, therefore, a key element in the global carbon cycle. Mapping of forest total biomass and change are needed for understanding carbon fluxes. Forest science has always aimed to reduce the effort of measuring AGB by developing allometric functions which deduce biomass from variables easier to measure, for instance diameter at breast height. An innovative approach shown in [1] proposes a height-to-biomass allometric relation which allows biomass estimation from RS systems capable of resolving forest height (LIDAR and polarimetric SAR interferometry (Pol-InSAR)) [2][3]:

$$
B=0.9 H^{1.58}
$$

where $B$ is above-ground biomass and $H$ forest height. In managed or disturbed forests however, height-biomass allometry can serve as a backbone of biomass estimation, but is not sufficient for reliable biomass estimations. This is due to a significant variance in stand densities at a given stand height. Thus, functions for the height-biomass relations need to be extended by incorporating forest structure parameters that correlate with stand density. The challenge is to identify appropriate forest structure variables that can be measured with SAR techniques. Among others, vertical reflectivity profiles are a very promising candidate.

Multi-baseline PolInSAR at a low electromagnetic frequency (e.g. L-band) allows to determine not only forest height, but also a full profile of the backscattered power (reflectivity) along the vertical dimension for each pixel, which depends on the horizontal and vertical structure of the vegetation. However, contrarily to forest height inversion, the physical interpretation of the vertical reflectivity profiles is still in a very early stage

This paper is organized as follows: first vertical biomass distribution is modelled and a vertical structure-to-biomass algorithm is developed; second, the transfer of this relation to SAR reflectivity profiles is tested and adapted. Finally, it is demonstrated that information obtained from SAR reflectivity profiles improves biomass estimations.

\section{VERTICAL STRUCTURE CHARACTERIZATION FOR BIOMASS ESTIMATION}

\subsection{Vertical biomass profiles}

Forest vertical structure can be investigated using vertical biomass profiles. In this study we model these profiles from ground inventory data from the test site Traunstein, which consists of 221 inventory plots with tree height, diameter at breast height (dbh) measurements and tree species information.

First, profiles are calculated summing up the biomass of all trees within a measurement plot $(0.05 \mathrm{ha})$ in $1 \mathrm{~m}$ steps along height. The obtained profile is then normalized to the 
total profile area, to obtain the final vertical biomass distribution.

The Legendre decomposition is used as a structure descriptor. Legendre coefficients are calculated according to (2).

$$
\begin{aligned}
& B(z)=\sum_{n} a_{n} P_{n}(z) \quad \text { where: } \\
& a_{n}=\frac{2 n+1}{2} \int_{-1}^{1} B(z) P(z) d z
\end{aligned}
$$

Where $a_{n}$ are the Legendre coefficients and $P_{n}$ the Legendre polynomials [4].


Figure 1: Biomass profiles for a high (black) and a low (blue) biomass level with $35 \mathrm{~m}$ forest height, reconstructed with 1 (upper left) to 4 (lower right) Legendre polynomials.

In [4] it was shown that a low number of Legendre polynomials (4) is sufficient to distinguish between different levels of biomass. In Figure 1 a step by step reconstruction with 1 to 4 Legendre polynomials is displayed for two profiles with the same height but with a high (black) and a low biomass (blue). When 4 polynomials are used, it is possible to clearly distinguish between two levels of biomass according to the profile shape.

\subsection{Structure ratio and biomass inversion}

The structure characterization developed here is based on the following principle. High biomass forest stands are characterized by frequencies connected to tree stems $\left(\mathrm{a}_{01}\right)$, approaching a homogeneous biomass distribution. However, for the same dominant height (H100) a stand with lower biomass, presents more gaps, and characterized by a higher proportion of frequencies connected to the crown compartments $\left(\mathrm{a}_{02}+\mathrm{a}_{03}+\mathrm{a}_{04}\right)$. Using the Legendre decomposition we propose a structure ratio which is based on the proportion between stem and crown frequencies (Eq. $3)$.

$$
S_{r a t}=\frac{\left|a_{01}\right|}{\left|a_{02}\right|+\left|a_{03}\right|+\left|a_{04}\right|}
$$

where $a_{0 n}$ are the normalized Legendre coefficients.
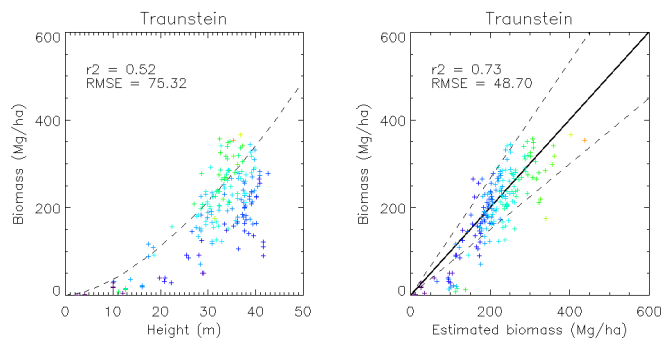

Figure 2: Height-to-biomass relation (right) and structure-to-biomass inversion (left) for Traunstein. Points are color-coded according to the structure ratio $S_{\text {rat }}$ from $\sim$ (blue) to 3 (red).

In Figure 2 - right, the height-to-biomass relation for Traunstein is displayed. Every point is color-coded according to the value of $S_{\text {rat }}$. Blue colors indicate a low $(\sim 0)$ ratio while red indicates a high ratio value (3). Above $15 \mathrm{~m}$, when forest structure begins to diversify, for a fixed height, points with low biomass (blue) have a lower value of $S_{r a t}$ when points with high biomass (red) have a higher value. $S_{\text {rat }}$ can then be used to modify the height to biomass allometry. A linear combination between the structure ratio and forest height has shown the best inversion results (Eq. $4)$.

$$
B=7 S_{\text {rat }}^{0.8} H^{0.85}
$$

\section{VERTICAL STRUCTURE FROM SAR}

In this work, airborne L-band data acquired with DLR's E-SAR during the TempoSAR 2008 campaign are used to investigate the capabilities of SAR tomography to invert forest biomass. Data were acquired on two days, one before a rain event and another after. Each dataset used is composed of 5 images (baselines 5, 10, 15, 25m).

The Capon method is applied on phase calibrated images to derive the vertical reflectivity profiles [6]. The resulting image has a horizontal spatial resolution of $\sim 12 \times 12 \mathrm{~m}$.

\section{BIOMASS INVERSION FROM SAR REFELECTIVITY PROFILES}

The biomass inversion algorithm proposed here comprises three stages: a profile based height estimation, an extinction correction and the application of an empirically derived structure-to-biomass allometric relation. 


\subsection{Height estimation}

In this work we try to retrieve biomass using only information provided by the reflectivity profiles. Therefore, forest height has been estimated from the reflectivity profile, similarly to the method shown in [5]. The main advantage of this approach is that the physically relevant features of the profile are preserved.
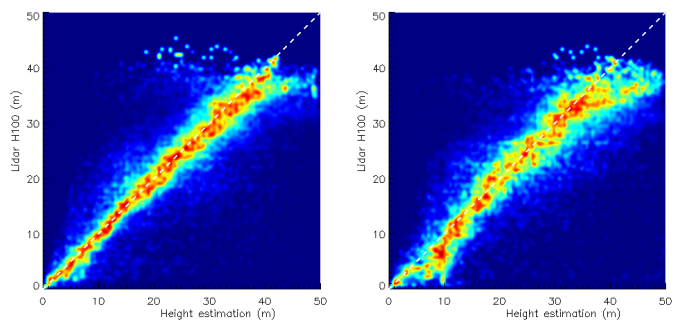

Figure 3: Height estimation vs. Lidar H100. Left: acquisition after rain; right: acquisition before rain; both in $\mathrm{HH}$ polarization.

Height detection is based on the distribution of the profile lobes, i.e. local profile maxima. It is possible that a vertical profile presents several physically relevant lobes. Height estimation from vertical reflectivity profiles requires distinguishing between physically relevant lobes and sidelobes. As the ground location is kwon [6] relevant side-lobes appear only above the physically relevant lobes. Then, the reflectivity profile is truncated when the power amplitude of the last lobe with a power larger than a $20 \%$ of the absolute maximum power, is below a $30 \%$.

The estimated height is plotted vs the LIDAR H100 in Figure 3. The height estimation in moist conditions (left), after rain, shows a better performance than in the acquisition before rain (right).

\subsection{Exponential correction}

In order to apply the structure ratio to the reflectivity profiles extinction needs to be corrected. We have observed that SAR reflectivity profiles the range of $S_{\text {rat }}$ is strongly reduced, due to the typical overemphasis of the power of the highest profile lobe induced by the signal extinction along forest height.

In order to compensate for this effect, an exponential correction is applied to every profile. The degree of correction is adapted in each profile, to profile height and the proportion between the largest (in terms of amplitude) and the lowest (closest to ground) physical profile lobes.

The reflectivity profile is, therefore, multiplied by an exponential curve $f(z)$ described through Eq. 5.

$$
f(z)=e^{-(a z-b)}
$$

where a and be are defined as:

$$
a=b-\operatorname{Ln}(0.01) / H ; b=\operatorname{Ln}\left(p i c_{r a t}\right),
$$

$\mathrm{H}$ is the profile height, $p i c_{\text {rat }}$ the ratio between the amplitude of the largest lobe $\left(A_{\max \text { pic }}\right)$ and the lowest lobe $\left(A_{\text {first pic }}\right)$ :

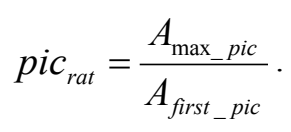

An example for the exponential correction of two profiles is displayed in Figure 4 (before correction (red) and after correction (green)).
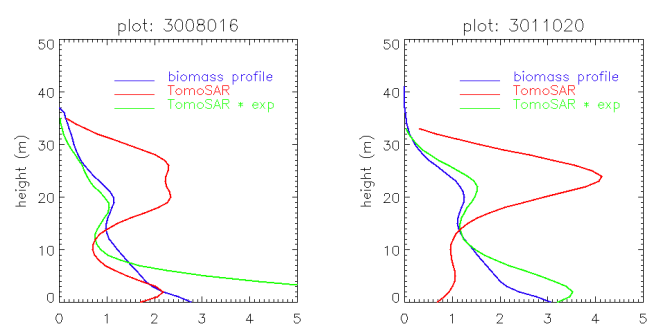

Figure 4: Exponential correction. Blue is the modelled biomass profile, red the original reflectivity profile and green the profile after exponential correction. All profiles are normalized.

\subsection{Biomass inversion}

Reflectivity profiles do not represent the exact vertical biomass distribution as obtained from ground measurements. L-band seems to be more sensitive to the leaf distribution in the crown volume than to the stem biomass.

In vertical biomass profiles, the low frequency coefficient $a_{01}$ is mainly driven by the biomass concentration in the tree stems and L-band is not so sensitive to them, then $S_{\text {rat }}$ obtained from the reflectivity profiles, is more dominated by the crown compartments. Nevertheless, crown volume distribution is strongly connected to tree density and biomass. As a consequence, a higher presence of biomass will be indicated by larger crown volumes along the profile and therefore a higher proportion of crown frequencies $\left(a_{02}\right.$, $\left.a_{03}, a_{04}\right)$.

Hence, the structure-to-biomass relation for SAR profiles is deduced as described by Eq. 7 .

$$
B=40 S_{r a t}^{-0.55} H^{0.85} \text {. }
$$



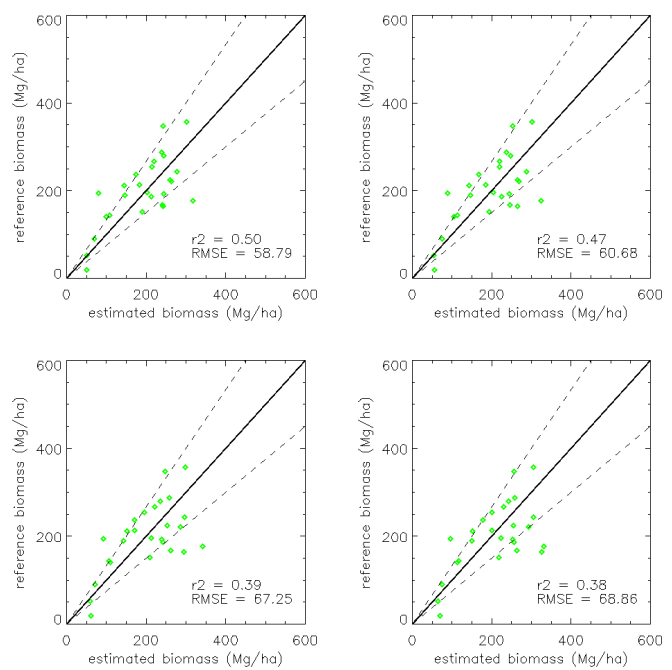

Figure 5: Biomass inversion using height-to-biomass allometry (Eq. 1); top, acquisition after rain and bottom acquisition before rain; left, $\mathrm{HH}$ and right, $\mathrm{HV}$.
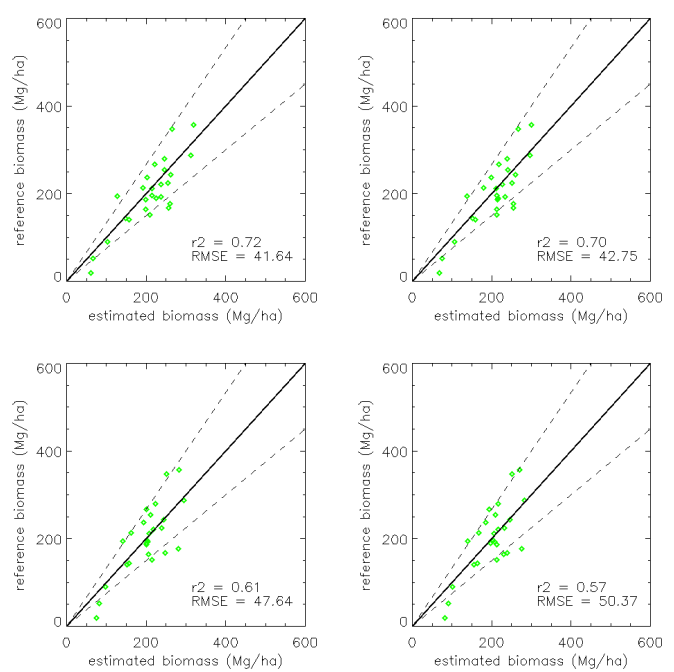

Figure 6: Biomass inversion using structure-to-biomass allometry (Eq. 7); top, acquisition after rain and bottom acquisition before rain; left, $\mathrm{HH}$ and right, $\mathrm{HV}$.

\subsection{First results}

In Figure 5 and Figure 6 inversion results for two sets of SAR data, before and after rain, in $\mathrm{HH}$ and $\mathrm{HV}$ are shown, using height-to biomass (Eq. 1) and structure-to-biomass (Eq. 6) allometry respectively.

In order to validate the biomass inversion results an interpolated map has been generated using the set of ground inventory plots. For validation homogenous stands, in terms of biomass, are used. The best inversion results are obtained with the acquisition after rain in $\mathrm{HH}$ polarization although
HV performs similarly. In the dry case, height estimation accuracy is reduced, affecting also the biomass estimation accuracy. Due to the higher penetration in this scenario the height estimation performance decreases and the profiles lobes are less clearly detectable. Nevertheless for all weather and polarization conditions we observe a constant improvement of $\sim 20 \%$ in the inversions scenarios with structure.

\section{CONCLUSIONS}

In this study we show that the structure-to-biomass allometry is able to improve the estimation of above-ground biomass and a vertical structure descriptor based on low frequency Legendre polynomials is sensitive to it. This ratio can be adapted to characterize vertical reflectivity profiles obtained from tomographic SAR techniques and an inversion algorithm, based on profile information only is proposed. The algorithm is tested for wet and dry forest conditions, as these can affect the shape of the reflectivity profiles.

\section{REFERENCES}

[1] T. Mette, K. Papathanassiou and I. Hajnsek, "Applying a common allometric equation to convert forest height from Pol-InSAR data to forest biomass", Proceedings of IGARSS, Anchorage, 2004

[2] K. Papathanassiou and S.R. Cloude, "Single-baseline Polarimetric SAR Interferometry", IEEE Transactions on Geoscience and Remote Sensing, pp. 2352-2363, 2001 ..

[3] R.N. Treuhaft et al., "Vegetation profiles in tropical forests from multibaseline interferometric synthetic aperture radar, field, and lidar measurements”, Journal of Geophysical Research. pp. 114, 2009

[4] A. C. Torano, F. Kugler, K. Papathanassiou, P. Biber and P. Pretzsch, "Biomass estimation as a function of vertical forest structure and forest height. Potential and limitations for Radar Remote Sensing”, Proceedings of EUSAR, Aachen, 2010.

[5] S. Tebaldini and F. Rocca, "Multibaseline Polarimetric SAR Tomography of a Boreal Forest at P- and LBands", IEEE Transactions on Geoscience and Remote Sensing, vol 50., no 1. pp. 2352-2363, 2012

[6] M. Pardini, A. Cantini, F. Lombardini and K. Papathanassiou, "3-D Structure of Forests: First Analysis of Tomogram Changes Due to Weather and Seasonal Effects at L-Band", Proc. of EUSAR, Berlin, Jun 2014 